\title{
Primal, dual and primal-dual partitions in continuous linear optimization
}

\section{MIGUEL A. GOBERNA ${ }^{1}$, MAXIM I. TODOROV ${ }^{2}$}

1 Dep. of Statistics and Oper. Res., University of Alicante, 03080 Alicante, Spain. e-mail: mgoberna@ua.es Research supported by DGES of Spain and FEDER of EU, Grant MTM200508572-C03-01

2 Dep. of Physics and Mathematics, UDLA, 72820 San Andrés Cholula, Puebla, Mexico. On leave from IMI-BAS, Sofia, Bulgaria. e-mail: maxim.todorov@udlap.mx Research partially supported by CONACyT of MX.Grant 44003

The date of receipt and acceptance will be inserted by the editor

\begin{abstract}
We associate with each natural number $n$ and each compact Hausdorff topological space $T$ the space of linear optimization problems with $n$ primal variables and index set $T$ (for the constraints) equipped with the topology of the uniform convergence. We consider three different partitions of this pseudometric space. The primal and the dual partitions are the result of classifying a given optimization problem and its dual as either inconsistent or bounded or unbounded, whereas the primal-dual partition is formed by the non-empty intersections of the elements of both partitions. The elements of the three partitions are neither open nor closed and their topological interiors are formed by those problems for which sufficiently small perturbations maintain the membership of the problem, i..e., the problems that are stable for the corresponding property. We prove that the stable problems are the same for the three partitions, concluding that most problems are stable in the three senses. This is done by completing the topological analysis of the primal-dual partition carried out in a previous paper of the authors.
\end{abstract}

Key words linear programming - linear semi-infinite programming - stability

Correspondence to: The above adresses 


\section{Introduction}

Let $n \in \mathbb{N}, n \geq 2$, and a non-empty compact Hausdorff topological space $T$ be given. We associate with each triple

$$
\pi:=(a, b, c) \in \Pi:=\mathcal{C}(T)^{n} \times \mathcal{C}(T) \times \mathbb{R}^{n}
$$

an optimization problem called primal,

$$
\begin{aligned}
& P: \text { Min } c^{\prime} x \\
& \text { s.t. } a_{t}^{\prime} x \geq b_{t}, \quad t \in T,
\end{aligned}
$$

with space of variables $\mathbb{R}^{n}$, and its (Haar's) dual problem

$$
\begin{aligned}
D: & \operatorname{Max} \\
\text { s.t. } & \sum_{t \in T} \lambda_{t} b_{t} \\
& \sum_{t \varepsilon T} \lambda_{t} a_{t}=c, \\
& \lambda_{t} \geq 0, t \in T,
\end{aligned}
$$

whose space of variables is the linear space of all the functions $\lambda: T \mapsto \mathbb{R}$ such that $\lambda_{t}=0$ for all $t \in T$ except maybe for a finite number of indices. This space is denoted by $\mathbb{R}^{(T)}$ (the space of generalized finite sequences) and its positive cone by $\mathbb{R}_{+}^{(T)}$. If $|T|<\infty$ and we consider $T$ equipped with the discrete topology, $P$ and $D$ are ordinary linear programming (LP) problem. Otherwise, $P$ and $D$ are continuous linear semi-infinite programming (LSIP) problems as far as either the number of variables or the number of constraints (but not both) is infinite. Interesting applications of continuous LSIP can be found in [7, Chapters 1-2], [6], and the references therein. $\Pi$ is called the parameters space, and it can be the result of all possible perturbations performed on a given continuous problem provided the structure of the problem is preserved. In particular, $\Pi:=\mathbb{R}^{n|T|+|T|+n}$ when $T$ is finite.

We denote by $v^{P}(\pi)\left(v^{D}(\pi)\right)$ the optimal value of $P(D)$, defining as usual $v^{P}(\pi)=+\infty\left(v^{D}(\pi)=-\infty\right.$, respectively) when the corresponding problem is inconsistent. A problem is bounded when its optimal value is a real number. Given $\pi:=(a, b, c) \in \Pi$, since $P$ can be either inconsistent (I) or bounded (B) or unbounded (U), we can classify $\pi$ in one of the elements of the (ternary) primal partition $\left\{\Pi_{I}^{P}, \Pi_{B}^{P}, \Pi_{U}^{P}\right\}$. Similarly, $\pi$ can be classified in one of the elements of the (ternary) dual partition $\left\{\Pi_{I}^{D}, \Pi_{B}^{D}, \Pi_{U}^{D}\right\}$. We also denote by $\Pi_{C}^{P}:=\Pi_{B}^{P} \cup \Pi_{U}^{P}\left(\Pi_{C}^{D}:=\Pi_{B}^{D} \cup \Pi_{U}^{D}\right)$ the sets of parameters with consistent primal problem (dual problem, respectively). The (ternary) primal-dual partition is formed by the non-empty pairwise intersections of the elements of the primal and the dual partitions. The elements of the primal-dual partition are codified as shown in Table 1, where the set in each cell is the intersection of the entries of its column and its row (e.g., $\Pi_{B I}:=\Pi_{B}^{P} \cap \Pi_{I}^{D}$, etc.): 


\begin{tabular}{|l||l|l|l|}
\cline { 2 - 4 } \multicolumn{1}{c||}{} & $\Pi_{I}^{P}$ & $\Pi_{B}^{P}$ & $\Pi_{U}^{P}$ \\
\hline \hline$\Pi_{I}^{D}$ & $\Pi_{I I}$ & $\Pi_{B I}$ & $\Pi_{U I}$ \\
\hline$\Pi_{B}^{D}$ & $\Pi_{I B}$ & $\Pi_{B B}$ & \\
\hline$\Pi_{U}^{D}$ & $\Pi_{I U}$ & & \\
\hline \multicolumn{4}{|c|}{ Table 1} \\
\end{tabular}

The null element of $\Pi$, denoted by $\theta$, belongs to $\Pi_{B B}=\Pi_{B}^{P} \cap \Pi_{B}^{D}$, the set of parameters with bounded associated problems. Each element of the primal-dual partition corresponds to a duality state ([1] and [11] have analyzed the role played by the duality states in LP and LSIP).

We consider $\Pi$ as a metric space equipped with the following distance: given two parameters $\pi^{1}=\left(a^{1}, b^{1}, c^{1}\right)$ and $\pi^{2}=\left(a^{2}, b^{2}, c^{2}\right)$,

$$
d\left(\pi^{1}, \pi^{2}\right):=\max \left\{\left\|c^{1}-c^{2}\right\|_{\infty}, \max _{t \in T}\left\|\left(\begin{array}{c}
a_{t}^{1} \\
b_{t}^{1}
\end{array}\right)-\left(\begin{array}{c}
a_{t}^{2} \\
b_{t}^{2}
\end{array}\right)\right\|_{\infty}\right\} .
$$

In fact, it can be easily seen that $\Pi$ is also a Banach space with the usual sup norm. Throughout the paper the elements of $\Pi$ will be distinguished by means of upperscripts, and the same (either as subscripts or as superscripts) applies for their corresponding objects: $\pi^{r}=\left(a^{r}, b^{r}, c^{r}\right), D_{r}, P_{r}$, and so on.

In general LSIP, the functions $a: T \mapsto \mathbb{R}^{n}$ and $b: T \mapsto \mathbb{R}$ are not necessarily continuous, so that the space of parameters is $\left(\mathbb{R}^{n}\right)^{T} \times \mathbb{R}^{T} \times \mathbb{R}^{n}$. Replacing "max" with "sup" in (1), we get a pseudometric on this space, which generates the topology of uniform convergence and for which our parameters space $\Pi$ is a topological subspace.

A mathematical programming problem is called ill-posed in the feasibility sense if arbitrarily small perturbations provide both consistent and inconsistent problems ([13], [5] and [2] give formulae for the distance to illposedness in ordinary LP, in conic LP, and in general LSIP, respectively). In terms of the primal (dual) partition, the set of well-posed problems in feasibility sense is then the union of topological interiors $\left(\operatorname{int} \Pi_{C}^{P}\right) \cup\left(\operatorname{int} \Pi_{I}^{P}\right)$ $\left(\left(\operatorname{int} \Pi_{C}^{D}\right) \cup\left(\operatorname{int} \Pi_{I}^{D}\right)\right.$, respectively). Thus, int $\Pi_{C}^{P}$ and $\operatorname{int} \Pi_{I}^{P}$ (int $\Pi_{C}^{D}$ and int $\Pi_{I}^{D}$ ) can be seen as the set of primal (dual) stable problems. These interiors have been characterized in [8], [7] and [9]. On the other hand, [12] defines a conic programming problem to be ill-posed (in primal-dual feasibility sense) when it lays on the boundary of the set of consistent problems whose corresponding dual is also consistent. This class of primal-dual illposed parameters is, in our setting, the boundary of $\Pi_{B B}$, whose interior was characterized in [14], whereas the interior of the remaining elements of the primal-dual partition have been characterized in ([10]).

Let us observe that the recent paper [4] provides characterizations of the interior, the boundary and the exterior of the sets $\Pi_{C}^{D}$ and $\Pi_{B B}$ in general LSIP in terms of the data. Obviously, these characterizations become sufficient conditions in the context of continuous LSIP. In this context, $\operatorname{int} \Pi_{B}^{P}$ coincides with the interior of the class of those $\pi \in \Pi$ such that the corresponding primal problem $P$ is solvable ([3, Theorem 1]). 
The main objective of this paper is the qualitative analysis of the elements (non-convex cones) of the three partitions, which turn out to be neither open nor closed. More in detail, the paper is organized as follows: Section 2 contains additional notation and some basic results to be used later. Section 3 completes the topological analysis of the primal-dual partition carried out in [10], determining the pairs of elements of the this partition, $\left(\Pi_{i}, \Pi_{j}\right)$, such that any parameter in $\Pi_{i}$ can be approached from $\Pi_{j}$. Finally, Sections 4 and 5 characterize the interior of the elements of the primal and the dual partitions (recall that int $\Pi_{I}^{P}$ and int $\Pi_{I}^{D}$ have been already characterized), respectively, showing that they are dense subsets in the corresponding class, i.e., that most problems are primal (dual) stable (as it happens with the primal-dual partition according to [10]).

\section{Preliminaries}

Let us introduce the necessary notation. $0_{p}$ denotes the null-vector in $\mathbb{R}^{p}$, the $j$ th element of the canonical basis of $\mathbb{R}^{p}$ is $e_{j}$. The Euclidean and the $l_{\infty}$ (or Chebyshev) norms (in any of the spaces $\mathbb{R}^{p}$ and $\mathcal{C}(T)$ ) are represented by $\|\cdot\|$ and $\|\cdot\|_{\infty}$, respectively. Given a non-empty set $X \subset \mathbb{R}^{p}$, conv $X$ and cone $X:=\mathbb{R}_{+}$conv $X$ denote the convex hull and the conical convex hull of $X$, respectively (it is also assumed that cone $\emptyset=\left\{0_{p}\right\}$ ). If $X$ is convex, $\operatorname{dim} X$ denotes its dimension. From the topological side, if $X$ is a subset of any topological space, $\operatorname{int} X, \operatorname{cl} X$ and $\mathrm{bd} X$ represent the interior, the closure and the boundary of $X$, respectively.

The next result on Chebyshev functional approximation is used frequently throughout the paper.

Lemma 1 Let $T$ be a compact Hausdorff space, $\bar{x} \in \mathbb{R}^{n}$ and $x \in \mathcal{C}(T)^{n}$. Then the following statements hold:

(i) If $0_{n} \neq \bar{x} \notin$ int cone $\{x(t), t \in T\}$, then for all $\varepsilon>0$ there exists $y \in \mathcal{C}(T)^{n}$ such that $\bar{x} \notin$ cl cone $\{y(t), t \in T\}$ and $\|y-x\|_{\infty}<\varepsilon$.

(ii) If $0_{n} \neq \bar{x} \in \operatorname{clcone}\{x(t), t \in T\}$ and $|T| \geq n$, then for all $\varepsilon>0$ there exists $y \in \mathcal{C}(T)^{n}$ such that $\bar{x} \in$ int cone $\{y(t), t \in T\}$ and $\|y-x\|_{\infty}<\varepsilon$.

Proof (i) Let $d \in \mathbb{R}^{n} \backslash\left\{0_{n}\right\}$ such that $d^{\prime} \bar{x} \leq 0$ and $d^{\prime} x(t) \geq 0$ for all $t \in T$.

Let $y:=x+\frac{\varepsilon d}{2\|d\|_{\infty}} \in \mathcal{C}(T)^{n}$. Obviously, $\|y-x\|_{\infty}<\varepsilon$. If $y(t)=0_{n}$ for some $t \in T$, then $d^{\prime} x(t)=-\frac{\varepsilon\|d\|^{2}}{2\|d\|_{\infty}}<0$. Thus $y(t) \neq 0_{n}$ for all $t \in T$. Let $\alpha:=\max _{t \in T}\|y(t)\|>0$.

Assume that $\bar{x} \in \operatorname{clcone}\{y(t), t \in T\}$. Let $\left\{\lambda^{r}\right\} \subset \mathbb{R}_{+}^{(T)}$ such that $\bar{x}=\lim \sum_{t \in T} \lambda_{t}^{r} y(t)$. Since $\left\{\sum_{t \in T} \lambda_{t}^{r}\right\} \subset \mathbb{R}_{+}$, we can assume without loss of generality the existence of $\lim \sum_{t \in T} \lambda_{t}^{r}($ possibly $+\infty)$. We have 


$$
\|\bar{x}\|=\left\|\lim \sum_{t \in T} \lambda_{t}^{r} y(t)\right\| \leq \alpha \lim \sum_{t \in T} \lambda_{t}^{r} .
$$

Then

$$
\begin{aligned}
d^{\prime} \bar{x}= & \lim \sum_{t \in T} \lambda_{t}^{r} d^{\prime} y(t)=\lim \sum_{t \in T} \lambda_{t}^{r} d^{\prime}\left(x(t)+\frac{\varepsilon d}{2\|d\|_{\infty}}\right) \\
& \geq\left(\lim \sum_{t \in T} \lambda_{t}^{r} d^{\prime}\right) \frac{\varepsilon d}{2\|d\|_{\infty}} \geq \frac{\varepsilon\|\bar{x}\|\|d\|^{2}}{2 \alpha\|d\|_{\infty}}>0,
\end{aligned}
$$

in contradiction with $d^{\prime} \bar{x} \leq 0$.

(ii) Under the assumption, if $0_{n} \neq \bar{x} \in \operatorname{cone}\{x(t), t \in T\}$, then for all $\varepsilon>0$ there exists $y \in \mathcal{C}(T)^{n}$ such that $\bar{x} \in$ int cone $\{y(t), t \in T\}$ and $\|y-x\|_{\infty}<\varepsilon([10$, Lemma $1($ iii) $])$. The conclusion is trivial if either $T$ is finite or $0_{n} \notin \operatorname{conv}\{x(t), t \in T\}$ because then cone $\{x(t), t \in T\}$ is closed. Thus we assume that $T$ is infinite and $0_{n} \in \operatorname{conv}\{x(t), t \in T\}$. The last assumption implies that $\left(0_{n}, 1\right) \in \operatorname{cone}\{(x(t), 1), t \in T\}$, with $(x, 1) \in$ $\mathcal{C}(T)^{n+1}$. Again by $\left[10\right.$, Lemma $1($ iii) $]$, there exists $\left(y, y_{n+1}\right) \in \mathcal{C}(T)^{n+1}$ such that $\left\|\left(y, y_{n+1}\right)-(x, 1)\right\|_{\infty}<\varepsilon$ and

$$
\left(0_{n}, 1\right) \in \operatorname{int} \text { cone }\left\{\left(y(t), y_{n+1}(t)\right), t \in T\right\} .
$$

Then $\|y-x\|_{\infty}<\varepsilon$ and $0_{n} \in$ int cone $\{y(t), t \in T\}$, so that cone $\{y(t), t \in T\}=$ $\mathbb{R}^{n}$ and $\bar{x} \in$ int cone $\{y(t), t \in T\}$ trivially holds.

Statements (i) and (ii) in Lemma 1 are strong versions of statements (ii) and (iii) in [10, Lemma 1], showing that two convex cones can replaced by their corresponding closures.

Next we recall some basic results (most of them valid for general LSIP) we need on continuous linear optimization (all the proofs can be found in [7]). We associate with $\pi=(a, b, c)$ the feasible (optimal) sets of $D$ and $P$, which are denoted by $\Lambda$ and $F$ ( $\Lambda^{*}$ and $F^{*}$, respectively), the first and second moment cones of $\pi, M:=$ cone $\left\{a_{t}, t \in T\right\}$ and $N:=$ cone $\left\{\left(a_{t}, b_{t}\right), t \in T\right\}$, and the characteristic cone, $K:=N+\mathbb{R}_{+}\left\{\left(0_{n},-1\right)\right\}$. If $\pi$ satisfies the Slater condition, i.e., there exists $\bar{x} \in \mathbb{R}^{n}$ such that $a_{t}^{\prime} \bar{x}>b_{t}$ for all $t \in T$, then $N$ and $K$ are closed. A geometrical condition equivalent to the Slater condition is $0_{n+1} \notin \operatorname{conv}\left\{\left(a_{t}, b_{t}\right), t \in T\right\}$. If $D$ is consistent and $K$ is closed, then $D$ is solvable. The existence theorem establishes that $P$ is consistent if and only if $\left(0_{n}, 1\right) \notin \mathrm{cl} N$ if and only if $\left(0_{n}, 1\right) \notin \operatorname{cl} K$.

The next two lemmas summarize well-known results on the primal and dual binary partitions $\left(\left\{\Pi_{C}^{P}, \Pi_{I}^{P}\right\}\right.$ and $\left.\left\{\Pi_{C}^{D}, \Pi_{I}^{D}\right\}\right)$ and the binary primaldual partition. The proofs can be found in [8, Theorem 3.1], [9, Theorem 5], and [10] (where $N$ has been replaced with $K$ in all the characterizations), respectively. 
Lemma 2 The following statements are true:

(i) $\pi \in \operatorname{int} \Pi_{C}^{P}$ if and only if $P$ satisfies the Slater condition.

(ii) $\pi \in \operatorname{int} \Pi_{I}^{P}$ if and only if $\left(0_{n}, 1\right) \in \operatorname{int} N$.

(iii) $\pi \in \operatorname{int} \Pi_{C}^{D}$ if and only if $c \in \operatorname{int} M$.

(iv) $\pi \in \operatorname{int} \Pi_{I}^{D}$ if and only if there exists $y \in \mathbb{R}^{n}$ such that $c^{\prime} y<0$ and $a_{t}^{\prime} y>0$ for all $t \in T$.

Lemma 3 The elements of the primal-dual partition satisfy the following statements:

(i) $\pi \in \Pi_{B B}$ if and only if $\left(0_{n}, 1\right) \notin \mathrm{cl} K$ and $c \in M$. In particular, $\pi \in$ int $\Pi_{B B}$ if and only if Slater condition holds and $c \in \operatorname{int} M$. Moreover, int $\Pi_{B B}$ is dense in $\Pi_{B B}$ if and only if $|T| \geq n$.

(ii) $\pi \in \Pi_{U I}$ if and only if $\left(0_{n}, 1\right) \notin \operatorname{cl} K, c \notin M$ and $(\{c\} \times \mathbb{R}) \cap \operatorname{cl} K=\emptyset$. In particular, $\pi \in \operatorname{int} \Pi_{U I}$ if and only if there exists $y \in \mathbb{R}^{n}$ such that $c^{\prime} y<0$ and $a_{t}^{\prime} y>0$ for all $t \in T$. Moreover, int $\Pi_{U I}$ is dense in $\Pi_{U I}$.

(iii) $\pi \in \Pi_{I U}$ if and only if $\left(0_{n}, 1\right) \in \operatorname{cl} K, c \in M$ and $\{c\} \times \mathbb{R} \subset K$. In particular, $\pi \in \operatorname{int} \Pi_{I U}$ if and only if $\left(0_{n}, 1\right) \in \operatorname{int} K$. Moreover, int $\Pi_{I U}$ is dense in $\Pi_{I U}$ if and only if $|T| \geq n+1$.

(iv) $\pi \in \Pi_{I I}$ if and only if $\left(0_{n}, 1\right) \in \operatorname{cl} K$ and $c \notin M$. Moreover, int $\Pi_{I I}=\emptyset$.

(v) $\pi \in \Pi_{B I}$ if and only if $\left(0_{n}, 1\right) \notin \operatorname{cl} K, c \notin M$ and $(\{c\} \times \mathbb{R}) \cap \operatorname{cl} K \neq \emptyset$.

In particular, $\Pi_{B I}=\emptyset$ if $|T|<\infty$. Moreover, int $\Pi_{B I}=\emptyset$.

(vi) $\pi \in \Pi_{I B}$ if and only if $\left(0_{n}, 1\right) \in \operatorname{cl} K, c \in M$ and $\{c\} \times \mathbb{R} \nsubseteq K$. In particular, $\Pi_{I B}=\emptyset$ if $|T|<\infty$. Moreover, int $\Pi_{I B}=\emptyset$.

(vii) $\bigcup \quad \operatorname{int} \Pi_{i}$ is a dense subset of $\Pi$.

$i \in\{B B, U I, I U\}$

Lemma 4 Let $\pi \in \Pi$. If $c \notin M$ and $0_{n} \notin \operatorname{conv}\left\{a_{t}, t \in T\right\}$, then $\pi \in$ $\operatorname{int} \Pi_{U I}$.

Proof $0_{n} \notin \operatorname{conv}\left\{a_{t}, t \in T\right\}$ implies that $M$ is closed and pointed.

Since $c \notin M$ and this convex cone is closed, there exists $y \in \mathbb{R}^{n}$ such that $y^{\prime} c<0$ and $y^{\prime} a_{t} \geq 0$ for all $t \in T$.

The pointedness of $M$ implies the existence of $w \in \mathbb{R}^{n}$ such that $w^{\prime} z>0$ for all $z \in M \backslash\left\{0_{n}\right\}$. In particular, we have $w^{\prime} a_{t}>0$ for all $t \in T$.

Taking $\mu>0$ such that $(y+\mu w)^{\prime} c<0$, and observing that $(y+\mu w)^{\prime} a_{t}>$ $y^{\prime} a_{t} \geq 0$ for all $t \in T$, we conclude that $\pi \in \operatorname{int} \Pi_{U I}$ by Lemma 3(ii).

\section{The primal-dual partition revisited}

Proposition 1 The elements of the primal-dual partition are neither open nor closed.

Proof For any $i \in\{B B, \ldots, I I\}$ we have $\left(0_{n}, 0,0_{n}\right) \in \Pi_{B B} \cap \operatorname{cl} \Pi_{i}$, so that $\Pi_{i}$ cannot be closed. Now consider the sequence $\left\{\left(\frac{e_{1}}{r}, 1, e_{1}\right)\right\} \subset \Pi_{B B}$. Since $\lim \left(\frac{e_{1}}{r}, 1, e_{1}\right)=\left(0_{n}, 1, e_{1}\right) \in \Pi_{I I}, \Pi_{B B}$ is non-closed either.

Given $i \in\{I I, B I, I B\}$, we know that int $\Pi_{i}=\emptyset$ (Lemma 3 ), so that $\Pi_{i}$ cannot be open. The same is true for $\Pi_{B B}$ because $\left(0_{n}, 0,0_{n}\right) \in \Pi_{B B} \cap \mathrm{cl} \Pi_{i}$, 
for all $i \neq B B$, and also for $\Pi_{I U}$ because $\left(0_{n}, 1,0_{n}\right) \in \Pi_{I U}$ and it is the limit of $\left\{\left(\frac{e_{1}}{r}, 1, \frac{e_{1}}{r}\right)\right\} \subset \Pi_{B B}$. Thus, only $\Pi_{U I}$ could be open.

Assume that $|T|<\infty$. Select $\bar{t} \in T$ and let $\left(a_{\bar{t}}, b_{\bar{t}}\right)=0_{n+1}$ and $\left(a_{t}, b_{t}\right)=$ $\left(e_{1}, 0\right)$ for all $t \in T \backslash\{\bar{t}\}$, and $c=e_{2}$. Then $\pi=(a, b, c) \in \Pi_{U I}$ because $c \notin M$ and $\left(0_{n}, 1\right) \notin N$, but $\pi \notin \operatorname{int} \Pi_{C}^{P}$ because Slater condition fails and, so, $\pi \notin \operatorname{int} \Pi_{U I}$.

Alternatively, we assume that $T$ is infinite. As a consequence of Urisohn's lemma (see [8]), there exists a sequence $\left\{t_{r}\right\} \subset T$, with $t_{r} \neq t_{k}$ if $r \neq k$ and a function $\varphi \in \mathcal{C}(T)$ such that $\varphi: T \rightarrow[0,1]$ and $\varphi\left(t_{r}\right)=2^{1-r}$. Since $T$ is compact there exists a convergent subsequence. Let $\bar{t}$ be its limits. By a continuity argument, $\varphi(\bar{t})=0$.

According to Lemma 3(ii), $\pi:=\left(\varphi, \varphi^{2}, 0_{n-1},-e_{1}\right) \in \Pi_{U I}$. Since $a_{\bar{t}}=0_{n}$, it does not exist $y \in \mathbb{R}^{n}$ such that $c^{\prime} y=-y_{1}<0$ and $a_{t}^{\prime} y>0$ for all $t \in T$. Then $\pi \notin \operatorname{int} \Pi_{U I}$ and so $\Pi_{U I}$ is not open.

As a consequence of Lemma 3(vii), any element, $\pi$, of some meager duality state (i.e., with empty interior) can be approached from $\bigcup_{i \in\{B B, U I, I U\}} \operatorname{int} \Pi_{i}$, i..e, by means primal-dual stable problems. Next we prove that $\pi$ can be approached from each non-meager duality state if either $\pi \in \Pi_{B I}$ or $\pi \in \Pi_{I B}$. The elements of $\Pi_{I I}$ are always accessible from $\Pi_{U I}$, and also from $\Pi_{I U}$ if $|T| \geq n+1$. The accessibility from $\Pi_{B B}$, and also from $\Pi_{I U}$ if $|T| \leq n$, cannot be guarantied, as the next two examples show.

Example 1 Let $T=\{1,2,3\}$ and $n=2$ and $\pi=(a, b, c)$ such that $c=(1,0)$, whereas $(a, b)$ defined in two different ways:

(a) $\left(a_{1}, b_{1}\right)=(0,1,1),\left(a_{2}, b_{2}\right)=(0,-1,1)$ and $\left(a_{3}, b_{3}\right)=(0,0,1)$. It is easy to see that $\pi \in \Pi_{I I}$. Consider the sequence $\left\{\pi^{r}\right\} \subset \Pi$ such that $\pi^{r}=\left(a^{r}, b^{r}, c^{r}\right)$, with $\left(a_{1}^{r}, b_{1}^{r}\right)=\left(\frac{1}{r}, 1,1\right),\left(a_{2}^{r}, b_{2}^{r}\right)=\left(\frac{1}{r},-1,1\right),\left(a_{3}^{r}, b_{3}^{r}\right)=$ $\left(\frac{1}{r}, 0,1\right)$ and $c^{r}=c$. Since $\left\{\pi^{r}\right\} \subset \Pi_{B B}$ and $\pi^{r} \rightarrow \pi, \pi \in \operatorname{cl} \Pi_{B B}$.

(b) $\left(a_{1}, b_{1}\right)=(0,0,1),\left(a_{2}, b_{2}\right)=(-1,1,1)$ and $\left(a_{3}, b_{3}\right)=(-1,-1,1)$. Obviously, $\pi \in \Pi_{I I}$. It can be realized that, if $\pi^{r}$ is a perturbation of $\pi$ such that $d\left(\pi^{r}, \pi\right)<\frac{1}{2}$ and $c^{r} \in M_{r}$, then necessarily $a_{1}^{r} \in\left\{x \in \mathbb{R}^{2} \mid x_{1}>0\right\}$, in which case $\left(0_{2}, 1\right) \in N_{r}$. Thus, it is impossible to approach $\pi$ from $\Pi_{B B}$. i.e., $\pi \notin \operatorname{cl} \Pi_{B B}$.

The finiteness of $T$ is not essential in this example: take an infinite compact Hausdorff space $S$ and aggregate to $S$ two isolated points, $t_{1}$ and $t_{2}$. Then $T:=S \cup\left\{t_{1}, t_{2}\right\}$ is a compact Hausdorff space too and, taking $\left(a_{t_{i}}, b_{t_{i}}\right)=\left(a_{i}, b_{i}\right), i=1,2$, and $\left(a_{t}, b_{t}\right)=\left(a_{3}, b_{3}\right)$, for all $t \in S$, we get the same conclusions as above.

Example 2 Let $T=\{1,2\}, n=2$ and $\pi=(a, b, c)$ such that $\left(a_{1}, b_{1}\right)=$ $(0,1,1),\left(a_{2}, b_{2}\right)=(0,-1,1)$ and $c=(1,0)$. We have $\pi \in \Pi_{I I}$ because $c \notin M$ and $\left(0_{2}, 1\right) \in N$. Let $\left\{\pi^{r}\right\} \subset \Pi_{I U}$ such that $\pi^{r} \rightarrow \pi$. Since $a_{i} \neq 0_{2}$, $i=1,2, a_{i}^{r} \neq 0_{2}, i=1,2$, for $r$ large enough. In this case, since $\left(0_{2}, 1\right) \in N_{r}$ implies that $M_{r}=$ cone $\left\{a_{i}^{r}\right\}, i=1,2$, and $d\left(c, M_{r}\right) \rightarrow 1$ as $r \rightarrow \infty$. Since 
$c^{r} \rightarrow c$, we have $d\left(c^{r}, M_{r}\right) \rightarrow 1$ and so $c^{r} \notin M_{r}$ (contradiction). Hence we cannot approach $\pi$ from $\Pi_{I U}$. So the inclusion $\Pi_{I I} \subset \operatorname{cl} \Pi_{I U}$ fails if $|T| \leq n$.

Theorem $1 \Pi_{i} \subset \operatorname{cl} \Pi_{j}, i \in\{B I, I B\}, j \in\{B B, U I, I U\}$. Moreover, $\Pi_{I I} \subset$ $\operatorname{cl} \Pi_{U I}$, and $\Pi_{I I} \subset \operatorname{cl} \Pi_{I U}$ if $|T| \geq n+1$.

Proof $\Pi_{I I} \subset \operatorname{cl} \Pi_{U I}$ : Let $\pi \in \Pi_{I I}$. Since $c \notin M$, there exists, $z \in \mathbb{R} \backslash\left\{0_{n}\right\}$ such that $c^{\prime} z \leq 0$ and $a_{t}^{\prime} z \geq 0$ for all $t \in T$. For each $r \in \mathbb{N}$, let $a^{r}:=a+\frac{z}{r}$, $b^{r}:=b, c^{r}:=c-\frac{z}{r}$, and $\pi^{r}:=\left(a^{r}, b^{r}, c^{r}\right)$. Since $z^{\prime} c^{r}=c^{\prime} z-\frac{\|z\|^{2}}{r}<0$ and $z^{\prime} a_{t}^{r}=a_{t}^{\prime} z+\frac{\|z\|^{2}}{r}>0$ for all $t \in T$, we have $\left\{\pi^{r}\right\} \subset \operatorname{int} \Pi_{U I} \subset \Pi_{U I}$, with $\pi^{r} \rightarrow \pi$.

$\Pi_{I I} \subset \operatorname{cl} \Pi_{I U}$ if $|T| \geq n+1:$ Let $\pi \in \Pi_{I I}$, i.e., $c \notin M$ and $\left(0_{n}, 1\right) \in \operatorname{cl} N$. By Lemma 1(iii), applied to $\left(0_{n}, 1\right)$ and $N=$ cone $\left\{\left(a_{t}, b_{t}\right), t \in T\right\}$, there exists $\left\{\left(a^{r}, b^{r}\right)\right\} \subset \mathcal{C}(T)^{n+1}$ such that $\left(0_{n}, 1\right) \in \operatorname{int}$ cone $\left\{\left(a_{t}^{r}, b_{t}^{r}\right), t \in T\right\}$, with $\left(a^{r}, b^{r}\right) \rightarrow(a, b)$. Taking $\pi^{r}:=\left(a^{r}, b^{r}, c^{r}\right), r=1,2, \ldots$, with $c^{r}=c$ for all $r$, we have $\left\{\pi^{r}\right\} \subset \operatorname{int} \Pi_{I U}$ and $\pi^{r} \rightarrow \pi$. Thus $\Pi_{I I} \subset \operatorname{clint} \Pi_{I U} \subset \operatorname{cl} \Pi_{I U}$.

For the remaining inclusions $\Pi_{i} \subset \mathrm{cl} \Pi_{j}, i \in\{B I, I B\}, j \in\{B B, U I, I U\}$, we can assume that $T$ is infinite (otherwise $\Pi_{B I}=\Pi_{I B}=\emptyset$ and we are done).

$\Pi_{B I} \subset \mathrm{cl} \Pi_{B B}$ : Let $\pi \in \Pi_{B I}$. This means that $c \notin M,\left(0_{n}, 1\right) \notin \operatorname{cl} K$ and $(\{c\} \times \mathbb{R}) \cap \operatorname{cl} K \neq \emptyset$. In that case $c \in(\operatorname{cl} M) \backslash M$ and there exists a sequence $\left\{c^{r}\right\} \subset M$ such that $c^{r} \rightarrow c$. Defining $\pi^{r}:=\left(a, b, c^{r}\right)$ for all $r \in \mathbb{N}$, we have $\left\{\pi^{r}\right\} \subset \Pi_{B B}$ because $c^{r} \in M_{r}=M$ and $\left(0_{n}, 1\right) \notin \operatorname{cl} K_{r}=\operatorname{cl} K$ for all $r \in \mathbb{N}$.

$\Pi_{B I} \subset \operatorname{cl} \Pi_{U I}$ : Let $\pi \in \Pi_{B I}$. Take a sequence $\left\{c^{r}\right\} \subset \mathbb{R}^{n} \backslash \operatorname{cl} M$ such that $c^{r} \rightarrow c$. Let $\pi^{r}:=\left(a, b, c^{r}\right)$ for all $r \in \mathbb{N}$, so that $\pi^{r} \rightarrow \pi$. Obviously, given $r \in \mathbb{N}, c^{r} \notin M_{r}=M,\left(0_{n}, 1\right) \notin \operatorname{cl} K_{r}=\operatorname{cl} K$ and $\left(\left\{c^{r}\right\} \times \mathbb{R}\right) \cap \operatorname{cl} K_{r}=\emptyset$ (otherwise there exists $\alpha \in \mathbb{R}$ such that $\left(c^{r}, \alpha\right) \in \operatorname{cl} K_{r}=\operatorname{cl} K$ and so $\left.c^{r} \in M\right)$.

$\Pi_{B I} \subset \mathrm{cl} \Pi_{I U}$ : We discuss two possible cases for $\pi \in \Pi_{B I}$.

(a) $0_{n+1} \in \operatorname{conv}\left\{\left(a_{t}, b_{t}\right), t \in T\right\}$, i.e., $\left(0_{n+1}, 1\right) \in$ cone $\left\{\left(a_{t}, b_{t}, 1\right), t \in T\right\}$. By Lemma 1(ii), there exists $\left\{\left(a^{r}, b^{r}, \varphi_{r}\right)\right\} \subset \mathcal{C}(T)^{n+2}$ such that $\left(0_{n+1}, 1\right) \in$ int cone $\left\{\left(a_{t}^{r}, b_{t}^{r}, \varphi_{r}(t)\right), t \in T\right\}$ and $\left\{\left(a^{r}, b^{r}, \varphi_{r}\right)\right\} \rightarrow(a, b, 1)$. Then $0_{n+1} \in$ int cone $\left\{\left(a_{t}^{r}, b_{t}^{r}\right), t \in T\right\}$ and $\left\{\left(a^{r}, b^{r}\right)\right\} \rightarrow(a, b)$. Defining $\pi^{r}:=\left(a^{r}, b^{r}, c^{r}\right)$, $r=1,2, \ldots$, with $c^{r}=c$ for all $r$, we get $\pi^{r} \rightarrow \pi$ and $\pi^{r} \in \operatorname{int} \Pi_{I U}$ because $0_{n+1} \in \operatorname{int} N_{r} \subset \operatorname{int} K_{r}$ implies that $N_{r}=\mathbb{R}^{n+1}$ so that $\left(0_{n}, 1\right) \in \operatorname{int} N_{r}$ trivially. Thus $\Pi_{B I} \subset \operatorname{clint} \Pi_{I U} \subset \operatorname{cl} \Pi_{I U}$.

(b) $0_{n+1} \notin \operatorname{conv}\left\{\left(a_{t}, b_{t}\right), t \in T\right\}$. In that case $N$ is a closed convex cone and, so, $(\{c\} \times \mathbb{R}) \cap N=(\{c\} \times \mathbb{R}) \cap \operatorname{cl} N \neq \emptyset$. This implies that $c \in M$ (contradiction).

$\Pi_{I B} \subset \operatorname{cl} \Pi_{B B}:$ Let $\pi \in \Pi_{I B}$, i.e., $c \in M,\left(0_{n}, 1\right) \in \operatorname{cl} N$ and $\{c\} \times \mathbb{R} \nsubseteq \subseteq$. The last two conditions imply that $\left(0_{n}, 1\right) \in(\operatorname{cl} N) \backslash N$. By Lemma 1(i) there exists a sequence $\left\{\left(a^{r}, b^{r}\right)\right\}$ such that $\left(a^{r}, b^{r}\right) \rightarrow(a, b)$ and $\left(0_{n}, 1\right) \notin$ cl cone $\left\{\left(a_{t}^{r}, b_{t}^{r}\right), t \in T\right\}$. Let $\lambda \in \mathbb{R}_{+}^{(T)}$ be such that $c=\sum_{t \in T} \lambda_{t} a_{t}$. Consider 


$$
\begin{aligned}
c^{r}:=\sum_{t \in T} \lambda_{t} a_{t}^{r} \text { and } \pi^{r}:=\left(a^{r}, b^{r}, c^{r}\right), r=1,2, \ldots \text { Since } \\
\left\|c^{r}-c\right\|_{\infty}=\left\|\sum_{t \in T} \lambda_{t}\left(a_{t}^{r}-a_{t}\right)\right\|_{\infty} \leq \sum_{t \in T} \lambda_{t}\left\|\left(a_{t}^{r}-a_{t}\right)\right\|_{\infty},
\end{aligned}
$$

we have $c^{r} \rightarrow c$ and so $\pi^{r} \rightarrow \pi$. On the other hand, $c^{r} \in M_{r}$ and $\left(0_{n}, 1\right) \notin$ cl $N_{r}$ for all $r \in \mathbb{N}$ imply that $\left\{\pi^{r}\right\} \subset \Pi_{B B}$. Hence $\Pi_{I B} \subset \operatorname{cl} \Pi_{B B}$.

$\Pi_{I B} \subset \operatorname{cl} \Pi_{U I}$ : Let $\pi \in \Pi_{I B}$. Take $\alpha \in \mathbb{R}$ such that $(c, \alpha) \in N$. If $N$ is closed, since $\left(0_{n}, 1\right) \in N$, we have $(c, \gamma) \in N$ for all $\gamma \geq 0$, so that $\{c\} \times \mathbb{R} \subset K$ (contradiction). Thus $N$ is not closed, $0_{n+1} \in \operatorname{conv}\left\{\left(a_{t}, b_{t}\right), t \in T\right\}$ and $0_{n} \in \operatorname{conv}\left\{a_{t}, t \in T\right\}$.

We prove now that $c \notin \operatorname{rint} M$. Assume the contrary, i.e., $c \in \operatorname{rint} M$. Let $d=\operatorname{dim} M$, in which case $\operatorname{dim} K=\operatorname{dim} N=d+1$. Since $c \in \operatorname{rint} M, c$ is a positive convex combination of some affinely independent set $\left\{c_{1}, \ldots, c_{d+1}\right\} \subset$ $M$. For each $i=1, \ldots, d+1$ there exists a scalar $\alpha_{i}$ such that $\left(c_{i}, \alpha_{i}\right) \in N \subset$ $\operatorname{cl} N$, so that $\left\{c_{i}\right\} \times \mathbb{R} \subset \operatorname{cl} K$. Then

$$
\{c\} \times \mathbb{R} \subset \operatorname{conv}\left\{\bigcup_{i=1, \ldots, d+1}\left(\left\{c_{i}\right\} \times \mathbb{R}\right)\right\} \subset \operatorname{cl} K,
$$

where $\operatorname{dim} \operatorname{conv}\left\{\bigcup_{i=1, \ldots, d+1}\left(\left\{c_{i}\right\} \times \mathbb{R}\right)\right\}=d+1$. Since

$$
\operatorname{rint} \operatorname{conv}\left\{\bigcup_{i=1, \ldots, d+1}\left(\left\{c_{i}\right\} \times \mathbb{R}\right)\right\} \subset \operatorname{rint} \operatorname{cl} K=\operatorname{rint} K \subset K,
$$

we get $\{c\} \times \mathbb{R} \subset K$ (contradiction).

Hence $c \in M \backslash(\operatorname{rint} M) \subset \operatorname{cl} M \backslash(\operatorname{rint} \operatorname{cl} M)$. Since $\operatorname{cl} M \neq \operatorname{rint} \operatorname{cl} M, \operatorname{cl} M$ cannot be a linear subspace and, denoting by $L$ the lineality space of cl $M$, we can write $\operatorname{cl} M=C+L$, where $C:=(\operatorname{cl} M) \cap L^{\perp}$ is a closed convex pointed cone. Let $w \in \operatorname{rint} C$ such that $w^{\prime} x>0$ for all $x \in C \backslash\left\{0_{n}\right\}$. Given $z \in \operatorname{cl} M$, we can write $z=x+y$, with $x \in C$ and $y \in L$. Then we have $w^{\prime} z=w^{\prime} x+w^{\prime} y=w^{\prime} x \geq 0$ because $y \in L$ and $w \in L^{\perp}$. In particular, $w \in M \backslash\left\{0_{n}\right\}$ and satisfies $w^{\prime} a_{t} \geq 0$ for all $t \in T$.

Let $\pi^{r}:=\left(a+\frac{w}{r}, b, c\right)$ for all $r \in \mathbb{N}$. If $0_{n} \in \operatorname{conv}\left\{a_{t}^{r}, t \in T\right\}$, there exists $\lambda^{r} \in \mathbb{R}_{+}^{(T)}$ such that $0_{n}=\sum_{t \in T^{r}} \lambda_{t}^{r} a_{t}^{r}$ and $\sum_{t \in T^{r}} \lambda_{t}^{r}=1$. Multiplying by $w$ we obtain the following contradiction: $0=\sum_{t \in T^{r}} \lambda_{t}^{r}\left(a_{t}^{\prime} w+\frac{\|w\|^{2}}{r}\right)>0$. Thus, $0_{n} \notin \operatorname{conv}\left\{a_{t}^{r}, t \in T\right\}$ for all $r \in \mathbb{N}$.

For each $r \in \mathbb{N}$, since $M_{r} \subset M$ and $c \notin \operatorname{int} M, c \notin \operatorname{int} M_{r}$. Now we define a sequence $\left\{\widehat{\pi}^{r}\right\}$ as follows: 
If $c \notin M_{r}$, take $\widehat{\pi}^{r}:=\pi^{r}$. Otherwise, since $c \in M_{r} \backslash\left(\right.$ int $\left.M_{r}\right)$, take $\widehat{\pi}^{r}=$ $\left(a^{r}, b^{r}, c^{r}\right)$, where $c^{r} \notin M_{r}$ satisfies $\left\|c^{r}-c\right\|_{\infty}<\frac{1}{r}$. Since $\widehat{\pi}^{r} \rightarrow \pi$ and $\widehat{\pi}^{r} \in \operatorname{int} \Pi_{U I}$ for all $r \in \mathbb{N}$ (by Lemma 4), we conclude that $\Pi_{I B} \subset \operatorname{cl} \Pi_{U I}$.

$\Pi_{I B} \subset \mathrm{cl} \Pi_{I U}$ : Let $\pi \in \Pi_{I B}$. As in the proof of $\Pi_{I B} \subset \mathrm{cl} \Pi_{B B}$, we have $\left(0_{n}, \overline{1}\right) \in(\mathrm{cl} N) \backslash N$. By Lemma $1(\mathrm{i})$ there exists a sequence $\left\{\pi^{r}\right\}$ such that $\left(0_{n}, 1\right) \in \operatorname{int} N_{r}$ for all $r \in \mathbb{N}$, so that $\left\{\pi^{r}\right\} \subset \operatorname{int} \Pi_{I U} \subset \Pi_{I U}$, with $\pi^{r} \rightarrow \pi$.

The proof is complete.

The accessibility of the meager elements of the primal-dual partition from the non-meager ones, when $T$ is infinite, is represented in an intuitive way in Figure 1 (actually $\Pi$ is an infinite dimensional linear space and $\Pi_{B B}, \ldots, \Pi_{I B}$ are infinite dimensional non-convex cones). The figure shows that $\Pi_{B I}$ and $\Pi_{I B}$ are accessible from $\Pi_{B B}, \Pi_{U I}$ and $\Pi_{I U}$ whereas $\Pi_{I I}$ is only accessible from $\Pi_{U I}$ and $\Pi_{I U}$. For this reason, $\Pi_{B I}$ and $\Pi_{I B}$ are represented on the "axis" that also contains elements of bd $\Pi_{j}, j \in$ $\{B B, U I, I U\}$, as the next example shows.

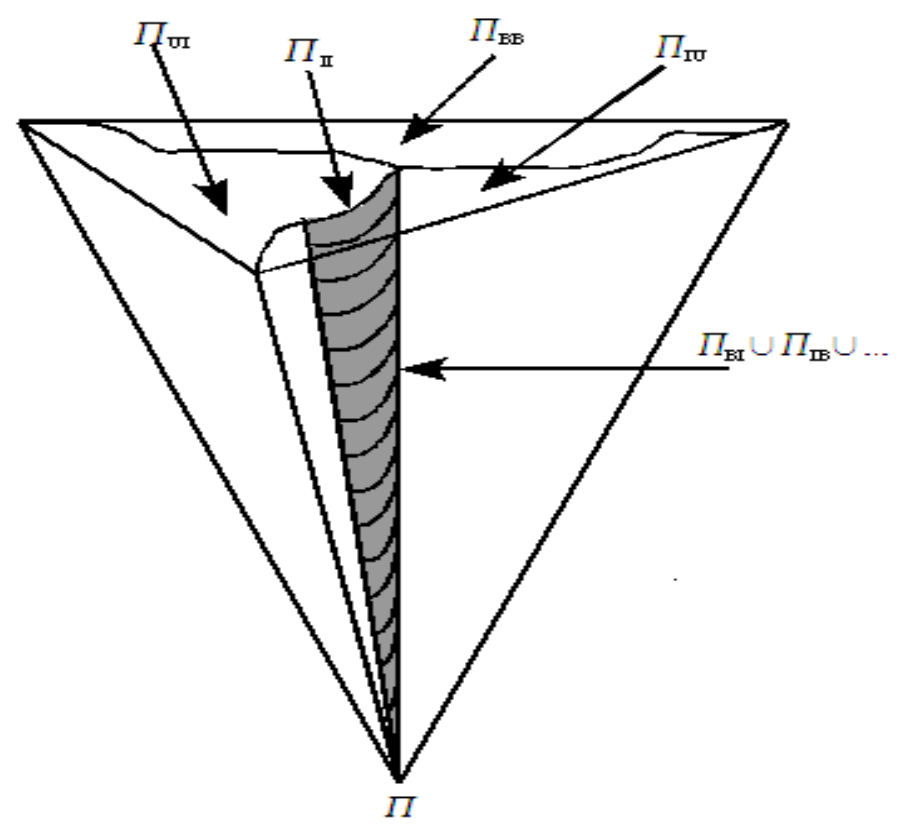

Figure 1: scheme of the partition of $\Pi$. 
Example 3 Let $T=\{1,2,3\}, n=2$ and $\pi=(a, b, c)$ such that $c=(0,-1)$ and

$$
\left(a_{t}, b_{t}\right)=\left\{\begin{array}{cc}
(1,1,1), & \text { if } t=1 \\
(-1,1,1), & \text { if } t=2, \\
(0,0,0), & \text { if } t=3 .
\end{array}\right.
$$

Since $(0,0,1) \in \operatorname{cl} K$ and $(\{c\} \times \mathbb{R}) \cap \operatorname{cl} K=\emptyset, \pi \in \Pi_{U I}$. We show next that $\pi$ is accessible from $\Pi_{B B}$ and $\Pi_{I U}$.

Given $r \in \mathbb{N}$, consider $\pi^{r}=\left(a^{r}, b^{r}, c^{r}\right)$ be such that $c^{r}=c,\left(a_{t}^{r}, b_{t}^{r}\right)=$ $\left(a_{t}, b_{t}\right), r=1,2$, and $\left(a_{3}^{r}, b_{3}^{r}\right)$ is defined in two different ways:

(a) $\left(a_{3}^{r}, b_{3}^{r}\right)=\left(0,-\frac{1}{r},-\frac{1}{r}\right)$. Then cl $K_{r} \subset\left\{x \in \mathbb{R}^{2} \mid x_{2} \geq x_{3}\right\}$, so that $(0,0,1) \notin$ cl $K_{r}$ and $c^{r} \in M_{r}=\mathbb{R}^{2}$. Thus $\pi^{r} \in \Pi_{B B}$.

(b) $\left(a_{3}^{r}, b_{3}^{r}\right)=\left(0,-\frac{1}{r}, 0\right)$. Since $\left\{\left(a_{i}^{r}, b_{i}^{r}\right), i=1,2,3 ;\left(0_{2},-1\right)\right\}$ is affinely independent and $0_{3}$ is positive convex combination of that vectors (with coefficients $\frac{1}{4+2 r}, \frac{1}{4+2 r}, \frac{1}{2+r}$ and $\frac{r}{2+r}$, respectively), $0_{3} \in \operatorname{int} K_{r}$. Then int $K_{r}=\mathbb{R}^{3}$ and we have $\pi^{r} \in \operatorname{int} \Pi_{I U}$.

Hence we have $\pi \in \bigcap_{j \in\{B B, U I, I U\}}$ bd $\Pi_{j}$ but $\pi \notin \bigcup_{i \in\{I I, B I, I B\}} \Pi_{i}$.

Figure 1 suggests that bd $\left[\bigcup_{j \in\{B B, U I, I U\}} \Pi_{j}\right] \subset\{\theta\} \cup\left[\bigcup_{i \in\{I I, B I, I B\}} \Pi_{i}\right]$.

Let us show that this is not always true.

Example \& Let $T=[0,1], n=2$ and $\pi=(a, b, c)$ such that $\left(a_{t}, b_{t}\right)=(0,0, t)$ for all $t \in T$ and $c=(0,0)$. We have $(0,0,1) \in \operatorname{cl} K$ and $c \in M=\left\{0_{2}\right\}$, so that $\pi \in \Pi_{I U}$ and $\pi \notin\{\theta\} \cup\left[\bigcup_{i \in\{I I, B I, I B\}} \Pi_{i}\right]$. Consider now, for $r \in \mathbb{N}$, $\pi^{r}=\left(a^{r}, b^{r}, c^{r}\right)$ such that $c^{r}=0_{2}$ and $\left(a_{t}^{r}, b_{t}^{r}\right)=\left(0, \frac{t^{2}}{r}, t\right), t \in T$. Then $\left(0_{2}, 1\right) \in \operatorname{cl} K_{r}, c^{r} \in M_{r}$ and $\left\{c^{r}\right\} \times \mathbb{R} \nsubseteq K_{r}$, so that $\pi^{r} \in \Pi_{I B}$. Hence $\pi \in \operatorname{cl} \Pi_{I B}$ and $\pi \in$ bd $\left[\bigcup_{j \in\{B B, U I, I U\}} \Pi_{j}\right]$.

\section{The primal partition}

Proposition 2 The elements of the primal partition are neither closed nor open.

Proof $\Pi_{I}^{P}$ and $\Pi_{U}^{P}$ cannot be closed because their boundary point $\left(0_{n}, 0,0_{n}\right)$ belongs to $\Pi_{B}^{P}$. This set is not closed because $\emptyset \neq \Pi_{I B} \subset \operatorname{clint} \Pi_{B B} \subset \operatorname{cl} \Pi_{B}^{P}$ and $\Pi_{I B} \subset \Pi_{I}^{P}$.

$\Pi_{B}^{P}$ cannot be open because its point $\left(0_{n}, 0,0_{n}\right) \in \operatorname{cl} \Pi_{I}^{P} \cap \operatorname{cl} \Pi_{U}^{P}, \Pi_{I}^{P}$ is not open because $\left(0_{n}, 1, e_{1}\right) \in \Pi_{I}^{P} \backslash\left(\operatorname{int} \Pi_{I}^{P}\right)$, and $\Pi_{U}^{P}$ is not open because it coincides with $\Pi_{U I}$. 
Theorem 2 The following statements hold:

(i) $\operatorname{int} \Pi_{I}^{P}=\operatorname{int} \Pi_{I U}$. Moreover, int $\Pi_{I}^{P}$ is dense in $\Pi_{I}^{P}$ if and only if $|T| \geq$ $n+1$.

(ii) $\operatorname{int} \Pi_{B}^{P}=\operatorname{int} \Pi_{B B}$. Moreover, int $\Pi_{B}^{P}$ is dense in $\Pi_{B}^{P}$ if and only if $|T| \geq n$.

(iii) int $\Pi_{U}^{P}=\operatorname{int} \Pi_{U I}$. Moreover, int $\Pi_{U}^{P}$ is dense in $\Pi_{U}^{P}$.

Thus, $\pi \in \Pi$ is primal stable if and only if it is primal-dual stable. Moreover, the primal stable parameters form an open dense subset of $\Pi$.

Proof (i) By Lemmas 2 and 3, we have

$$
\pi \in \operatorname{int} \Pi_{I}^{P} \Leftrightarrow\left(0_{n}, 1\right) \in \operatorname{int} K \Leftrightarrow \pi \in \operatorname{int} \Pi_{I U} .
$$

If $\operatorname{int} \Pi_{I}^{P}$ is dense in $\Pi_{I}^{P} \neq \emptyset$, then $\operatorname{int} \Pi_{I}^{P}=\operatorname{int} \Pi_{I U} \neq \emptyset$, and this implies $|T| \geq n+1$.

Conversely, assume that $|T| \geq n+1$. Since $\Pi_{I}^{P}=\Pi_{I U} \cup \Pi_{I I} \cup \Pi_{I B}$, $\operatorname{int} \Pi_{I}^{P}=\operatorname{int} \Pi_{I U}$ and $\operatorname{int} \Pi_{I U}$ is dense in $\Pi_{I U}$, it is enough to show that $\Pi_{I I} \subset \operatorname{cl} \Pi_{I U}$ and $\Pi_{I B} \subset \mathrm{cl} \Pi_{I U}$, but this was proved in Theorem 1 .

(ii) Since $\Pi_{B}^{P}=\Pi_{B B} \cup \Pi_{B I}$, int $\Pi_{B B} \subset \operatorname{int} \Pi_{B}^{P}$ and we must prove the reverse inclusion.

Let $\pi \in \operatorname{int} \Pi_{B}^{P}$. Then $\pi \in \operatorname{int} \Pi_{C}^{P}$ and so, by Lemma 2,

$$
0_{n+1} \notin \operatorname{conv}\left\{\left(a_{t}, b_{t}\right), t \in T\right\} \text {, }
$$

and this implies that $N$ is closed. Let $\varepsilon>0$ be such that $\pi^{1} \in \Pi_{B}^{P}$ if $d\left(\pi^{1}, \pi\right)<\varepsilon$. Taking an arbitrary $c^{1} \in \mathbb{R}^{n}$ such that $\left\|c^{1}-c\right\|_{\infty}<\varepsilon, \pi^{1}:=$ $\left(a, b, c^{1}\right) \in \Pi_{B}^{P}$. Hence, by Lemma 3 , either $\pi^{1} \in \Pi_{B B}$, with $c^{1} \in M_{1}$, or $\pi^{1} \in \Pi_{B I}$, in which case $\left(\left\{c^{1}\right\} \times \mathbb{R}\right) \cap N_{1} \neq \emptyset$, and we have also $c^{1} \in M_{1}$. We have shown that $c \in \operatorname{int} M$. This, together with (2), implies that $\pi \in$ int $\Pi_{B B}$, by Lemma $3(\mathrm{i})$.

If int $\Pi_{B}^{P}$ is dense in $\Pi_{B}^{P} \neq \emptyset$, then $\operatorname{int} \Pi_{B}^{P}=\operatorname{int} \Pi_{B B} \neq \emptyset$, and this implies $|T| \geq n$.

Now we assume $|T| \geq n$. Since $\Pi_{B}^{P}=\Pi_{B B} \cup \Pi_{B I}$, int $\Pi_{B}^{P}=\operatorname{int} \Pi_{B B}$, int $\Pi_{B B}$ is dense in $\Pi_{B B}$, and $\Pi_{B I} \subset \mathrm{cl} \Pi_{B B}$ (from Theorem 1), we conclude that int $\Pi_{B}^{P}$ is dense in $\Pi_{B}^{P}$.

(iii) It is trivial because $\Pi_{U}^{P}=\Pi_{U I}$.

\section{The dual partition}

Proposition 3 The elements of the dual partition are neither closed nor open.

Proof Since

$$
\left(0_{n}, 0,0_{n}\right) \in\left(\operatorname{cl} \Pi_{I}^{D}\right) \cap\left(\operatorname{cl} \Pi_{U}^{D}\right) \cap \Pi_{B}^{D},
$$


$\operatorname{cl} \Pi_{I}^{D}$ and $\operatorname{cl} \Pi_{U}^{D}$ are non-closed. On the other hand, $\emptyset \neq \Pi_{B I} \subset \operatorname{cl} \operatorname{int} \Pi_{B B} \subset$ cl $\Pi_{B}^{D}$ and $\Pi_{B I} \subset \Pi_{I}^{D}$, so that $\Pi_{B}^{D}$ is non-closed either.

$\Pi_{B}^{D}$ is not open as a consequence of (3) and the same applies to $\Pi_{U}^{D}$ because it coincides with $\Pi_{I U}$. Concerning $\Pi_{I}^{D}$, consider the sequence $\pi^{r}:=$ $\left(\frac{e_{1}}{r}, 0, e_{1}\right), r=1,2 \ldots$, with $\left\{\pi^{r}\right\} \subset \Pi_{B}^{D}$. Since $\pi^{r} \rightarrow \pi=\left(0_{n}, 0, e_{1}\right) \in \Pi_{I}^{D}$, this set is not open.

Theorem 3 The following statements hold:

(i) int $\Pi_{I}^{D}=\operatorname{int} \Pi_{U I}$. Moreover, int $\Pi_{I}^{D}$ is dense in $\Pi_{I}^{D}$.

(ii) int $\Pi_{B}^{D}=\operatorname{int} \Pi_{B B}$. Moreover, int $\Pi_{B}^{D}$ is dense in $\Pi_{B}^{D}$.

(iii) int $\Pi_{U}^{D}=\operatorname{int} \Pi_{I U}$. Moreover, int $\Pi_{U}^{D}$ is dense in $\Pi_{U}^{D}$ if and only if $|T| \geq n+1$.

Thus, $\pi \in \Pi$ is dual stable if and only if it is primal-dual stable. Moreover, the dual stable parameters form an open dense subset of $\Pi$.

Proof (i) The first statement is a straightforward consequence of Lemma 3.

Now consider the following partition of $\Pi_{I}^{D}: \Pi_{I}^{D}=\Pi_{U I} \cup \Pi_{I I} \cup \Pi_{B I}$. We know that int $\Pi_{I}^{D}=\operatorname{int} \Pi_{U I}$, int $\Pi_{U I}$ is dense in $\Pi_{U I}, \Pi_{I I} \subset \operatorname{cl} \Pi_{U I}$, and $\Pi_{B I} \subset \operatorname{cl} \Pi_{U I}$ (recall Theorem 1). Hence, int $\Pi_{I}^{D}$ is dense in $\Pi_{I}^{D}$.

(ii) For the first statement we have just to prove that int $\Pi_{B}^{D} \subset \operatorname{int} \Pi_{B B}$. Let $\pi \in \operatorname{int} \Pi_{B}^{D}$.

By Lemma 2 and the inclusion $\Pi_{B}^{D} \subset \Pi_{C}^{D}, c \in \operatorname{int} M$.

Now we assume that the Slater condition fails, i.e., $0_{n+1} \in \operatorname{conv}\left\{\left(a_{t}, b_{t}\right), t \in T\right\}$.

Let $\lambda \in \mathbb{R}_{+}^{(T)}$ be such that $0_{n+1}=\sum_{t \in T} \lambda_{t}\left(a_{t}, b_{t}\right)$ and $\sum_{t \in T} \lambda_{t}=1$, and let $\gamma \in \mathbb{R}_{+}^{(T)}$ be such that $c=\sum_{t \in T} \gamma_{t} a_{t}$.

Consider $\pi^{r}:=\left(a, b+\frac{1}{r}, c\right), r=1,2, .$. Obviously, $\pi^{r} \rightarrow \pi$.

Given $r \in \mathbb{N}, K_{r}$ contains the sequence

$$
\begin{aligned}
& \sum_{t \in T}\left(\gamma_{t}+r^{2} \lambda_{t}\right)\left(\begin{array}{c}
a_{t} \\
b_{t}+\frac{1}{r}
\end{array}\right) \\
& =\sum_{t \in T} \gamma_{t}\left(\begin{array}{c}
a_{t} \\
b_{t}+\frac{1}{r}
\end{array}\right)+r^{2} \sum_{t \in T} \lambda_{t}\left(\begin{array}{c}
a_{t} \\
b_{t}+\frac{1}{r}
\end{array}\right) \\
& =\left(\sum_{t \in T} \gamma_{t} b_{t}+\frac{1}{r} \sum_{t \in T} \gamma_{t}+r\right) \text {, }
\end{aligned}
$$

where $\lim \left(\sum_{t \in T} \gamma_{t} b_{t}+\frac{1}{r} \sum_{t \in T} \gamma_{t}+r\right)=+\infty$. Since $\{c\} \times \mathbb{R} \subset K_{r}, \pi^{r} \in \Pi_{I U}=$ $\Pi_{U}^{D}$, by Lemma 3 , contradicting $\pi \in \operatorname{int} \Pi_{B}^{D}$. Hence Slater condition holds.

Then $\pi \in \operatorname{int} \Pi_{B B}$ by Lemma $3(\mathrm{i})$.

Since $\Pi_{B}^{D}=\Pi_{B B} \cup \Pi_{I B}$, int $\Pi_{B}^{D}=\operatorname{int} \Pi_{B B}$, int $\Pi_{B B}$ is dense in $\Pi_{B B}$, and $\Pi_{I B} \subset \mathrm{cl} \Pi_{B B}$ (from Theorem 1), we conclude that int $\Pi_{B}^{D}$ is dense in $\Pi_{B}^{D}$

(iii) It is consequence of the identity $\Pi_{U}^{D}=\Pi_{I U}$. 


\section{References}

1. Ben-Israel, A., Charnes, A. and Kortanek, K.O., 1969, Duality and asymptotic solvability over cones. Bull. Amer. Math. Soc., 75, 318-324.

2. Cánovas, M.J., López, M.A., Parra, J. and Toledo, F.J., 2005, Distance to ill-posedness and consistency value of linear semi-infinite inequality systems. Math. Programming (Ser. A), 103, 95-126.

3. Cánovas, M.J., López, M.A., Parra, J. and Toledo, F.J., 2006, Ill-posedness with respect to the solvability in linear optimization. Linear Algebra Appl., 416, 520-540.

4. Cánovas, M.J., López, M.A., Parra, J. and Toledo, F.J., 2006, Distance to solvability / unsolvability in linear optimization, SIAM J. Optim., 16, 629649.

5. Epelman, M. and Freund, R.M., 2002, A new condition measure, preconditioners, and relations between different measures of conditioning for conic linear systems. SIAM J. Optim., 12, 627-655.

6. Goberna, M.A., 2005, Linear semi-infinite optimization: recent advances. In: A. Rubinov and V. Jeyakumar (Eds.) Continuous Optimization: Current Trends and Applications (New York: Springer-Verlag).

7. Goberna, M.A. and López, M.A., 1998, Linear Semi-Infinite Optimization (Chichester: John Wiley \& Sons).

8. Goberna, M.A., López, M.A. and Todorov, M. I., 1996, Stability theory for linear inequality systems. SIAM J. Matrix Anal. Appl., 17, 730-743.

9. Goberna, M.A., López, M.A. and Todorov, M.I., 2001, On the stability of the feasible set in linear optimization. Set-Valued Analysis, 9, 75-99

10. Goberna, M.A. and Todorov, M.I., Primal-dual stability in continuous linear optimization, Math. Programming (Ser. B), to appear.

11. Kortanek, K.O. and Zhang, Q., 2001, Perfect duality in semi-infinite and semidefinite programming. Math. Programming (Ser. A), 91, 127-144.

12. Nunez, M.A., 2002, A characterization of ill-posed data instances for convex programming, Math. Programming (Ser. A), 91, 375-390

13. Renegar, J., 1995, Linear programming, complexity theory and elementary functional analysis. Math. Programming (Ser. A), 70, 279-351.

14. Todorov, M.I., 1985-86, Generic existence and uniqueness of the solution set to linear semi-infinite optimization problems, Numer. Funct. Anal. Optim., 8, 541-556. 